\title{
ECGi Metrics in Atrial Fibrillation Dependency on Epicardium Segmentation
}

\author{
Ana González-Ascaso, Rubén Molero, Andreu M Climent, María S Guillem \\ ITACA Institute, Universitat Politècnica de València, Valencia, Spain
}

\begin{abstract}
Noninvasive electrocardiographic imaging (ECGi) is a useful tool that can be used to guide ablation procedures in atrial fibrillation (AF patients). Most ECGi resolutions are based on the Boundary Element Method, and thus application of Green's theorem, that requires that electrical sources reside inside a closed volume.

The objective of this work is to quantify the error in atrial fibrillation metrics than can be expected if two volumes are segmented for the atria instead of just one.

Our results show that segmenting the atria of the patients into two volumes instead of one does impact on rotor-related AF metrics that can be derived from ECGi whereas dominant-frequency metrics are less affected.
\end{abstract}

\section{Introduction}

Electrocardiographic imaging is a noninvasive method that can be used to detect and analyse certain cardiac arrythmias through the obtention of high-resolution images of the electrical activity of the heart. The result is dependent on the torso and heart's 3D geometry, as well as the electrical recordings from the patient. This technique can be used to guide surgical procedures such as ablations in atrial fibrillation. Consequently, the correct identification of the arrythmias in the atria is determinant on the result of the procedure.

The Boundary Element method (BEM) is one of the most used numerical computational methods in the resolution of ECGi problems. BEM is known to be applicable when Green's theorem can be applied which requires that electrical sources reside inside a closed volume [1].

On the other side, segmentation from magnetic resonance imaging allows us to obtain personalize atria geometries from patients and could be carried out in different ways: left and right atrium together in a single volume or separated into two volumes. The effect of this different segmentation when the geometry is used to calculate ECGi trough BEM is unknown and could have high clinical relevance. The aim of this study is to investigate the consequences if two volumes are segmented instead of just one and quantify the error using atrial fibrillation metrics: frequency and rotor related $\mathrm{AF}$ metrics.

\section{Methods}

We segmented 11 different atria from magnetic resonance imaging in 2 different ways: both atrium conforming a single volume and left and right atrium separated into 2 volumes, obtaining two different geometrical models of the same heart. The segmenting tool used to obtain the atria's 3D geometry was Itksnap-1.8.0 ITK-SNAP 1.8 and the models were cleaned trough Meshmixer software [2].

The torso's 3D models were generated from video recordings by using a camara rotating $360^{\circ}$ around the patient. Frames were obtained from the video using VLC media player and proceed with different applications: VisualSFM, MeshLab and Meshmixer. Atria were located into the torso by using information from the magnetic resonance images.

Electrical recordings during adenosine administration were acquired using 54 surface electrodes located on the patient's torso [3]. The BSPM signals were divided into segments of 4 seconds of duration, filtered to eliminate noise with a band-pass filter between 2 and $45 \mathrm{~Hz}$ after Principal Component Analysis (PCA) approach was used to cancel ventricular information (QRST segment) if present in spite of the adenosine administration [4].

Using the processed electrical information of each patient and the 3D geometrical model consisting of the torso and the atria placed inside, the inverse problem was calculated by using zero-order Tikhonov regularization and L-curve optimization to obtain the inverse computed electrograms (ECGi).

After that, metrics that quantify dominant frequencies such as: ECGi High Dominant Frequency (HDF) (Hz), ECGi HDF Extension (\%), ECGi Median Dominant Frequencies (DF) (Hz), ECGi Minimum DF (Hz), ECGi HDF-median DF (Hz), ECGi Median DF extension (\%); and metrics that measure the presence of rotors: ECGi Singular Points (SP) per second, ECGi Rotors per second, ECGi Mean Time with Rotors (\%), ECGi Mean Rotor Duration (s) and ECGi SP in HDF zone were measured [5]. Atrial maps representing dominant frequency and rotor histogram were also calculated to support numerical results 
related with the calculation of the metrics described before.

\section{Results and Discussion}

Having obtained the signals and tridimensional geometries and calculated the AF metrics as described in Methods section, we studied the difference between the two segmentation groups (joined atria and separated atria) with a paired Student's t test.

The highest dominant frequency (DF) was found to be similar when calculated with two atria volumes and just one (9.36 vs. $9.31 \mathrm{~Hz}, \mathrm{p}>0.05)$ and same results were observed for the rest of metrics related with the quantification of dominant frequencies as shown in Figure 1 . The $\mathrm{p}$-values calculated for these frequency metrics are also presented in Table 1 .

The number of rotors per second, as well as singular points were higher when measured on separate atria $(8.32 \mathrm{e}+03$ vs. $8.98 \mathrm{e}+03, \mathrm{p}<0.05 ; 118.82$ vs. 137.52 , $\mathrm{p}<0.05$ respectively) while the mean rotor duration was higher in the single-volume atria (0.063 vs. $0.06 \mathrm{p}<\sim 0.05)$. The results for the rest of rotor-related metrics are shown in Table 2.

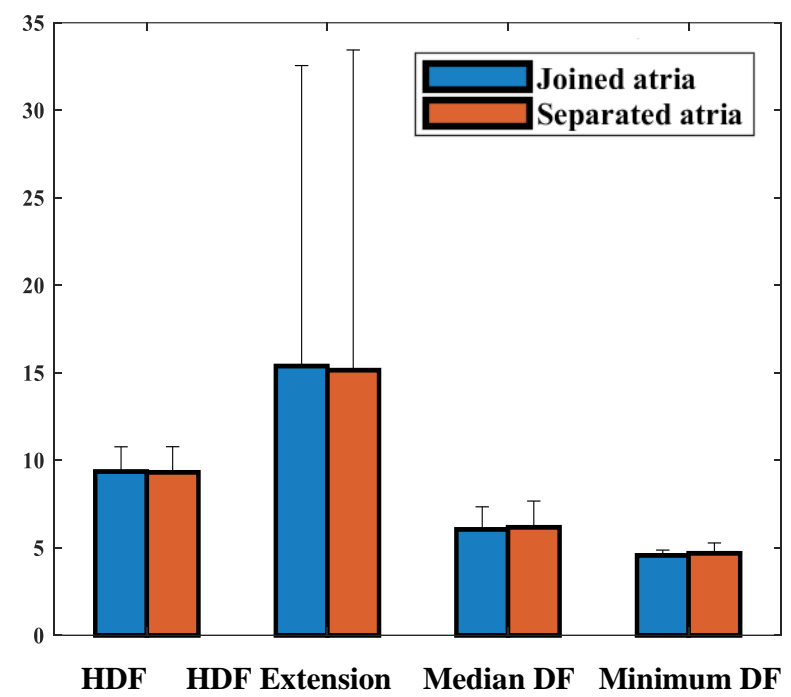

Figure 1. Mean and deviation of dominant frequency related AF metrics for different types of atria segmentation.

\begin{tabular}{ll}
\hline $\begin{array}{l}\text { Dominant Frequency } \\
\text { related AF metric }\end{array}$ & P value \\
\hline HDF (Hz) & 0.44 \\
HDF Extension (\%) & 0.83 \\
Median DF (Hz) & 0.23 \\
Minimum DF (Hz) & 0.25 \\
HDF-Median DF (Hz) & 0.13 \\
Median DF Extension (\%) & 0.54 \\
\hline
\end{tabular}

Table 1. P-value obtained in the comparation of frequency related metrics.

\begin{tabular}{|c|c|c|c|}
\hline Rotor-related metric & $\begin{array}{l}\text { Mean } \\
\text { Joined } \\
\text { Atria }\end{array}$ & $\begin{array}{l}\text { Mean } \\
\text { Sep. } \\
\text { Atria }\end{array}$ & P val. \\
\hline $\begin{array}{l}\text { Singular points per } \\
\text { second }\end{array}$ & $8.32 \mathrm{e}+03$ & $8.98 \mathrm{e}+03$ & 0.007 \\
\hline Rotors per second & 118.82 & 137.52 & $5.89 \mathrm{e}-4$ \\
\hline $\begin{array}{l}\text { Mean Time with } \\
\text { rotors }(\%)\end{array}$ & 92.81 & 94.28 & 0.28 \\
\hline $\begin{array}{l}\text { Mean rotor duration } \\
\text { (s) }\end{array}$ & 0.063 & 0.06 & 0.05 \\
\hline $\begin{array}{l}\text { Singular points in } \\
\text { High Dominant } \\
\text { Frequency zone }\end{array}$ & $2.63 e+03$ & $3.10 \mathrm{e}+03$ & 0.15 \\
\hline
\end{tabular}

Table 2. Comparation of the mean value obtained for the rotor related metrics for both segmentations: one volume (joined atria) and two volumes (separated atria) and the respective $\mathrm{p}$-value.

The results show that there are not statistical differences when frequency metrics are calculated for joined atria and separated atria since the p-values are in all cases bigger than 0.05 (Table 1). On the other hand, the paired Student's t test for the rotor-related AF metrics show different results for rotor-related metrics as it can be seen in Table 2. Singular points per second and Rotors per second show statistical differences between segmentation groups while the rest present a p-value equal or higher than 0.05 .

To further understand the results, some maps were represented. Dominant frequency maps show the distribution of dominant frequencies histogram in the atria, in other words, where the dominant frequencies are placed along the atria. These maps didn't show significant differences when left and right atrium are segmented in one volume or in two volumes, according with numerical results showed before. (Figure 2, A). Besides that, as it can be observed in Figure 2.B, rotor-related maps which consist of the quantification of singular points in the atria, present considerable differences between the segmentation groups. It can be observed that the number of singular points is higher when the atria are segmented conforming two volumes, which is the same result that can be observed in Table 2 for the number of singular point per second and rotors per second.

According to our results atria maps supported numerical results in both cases. 


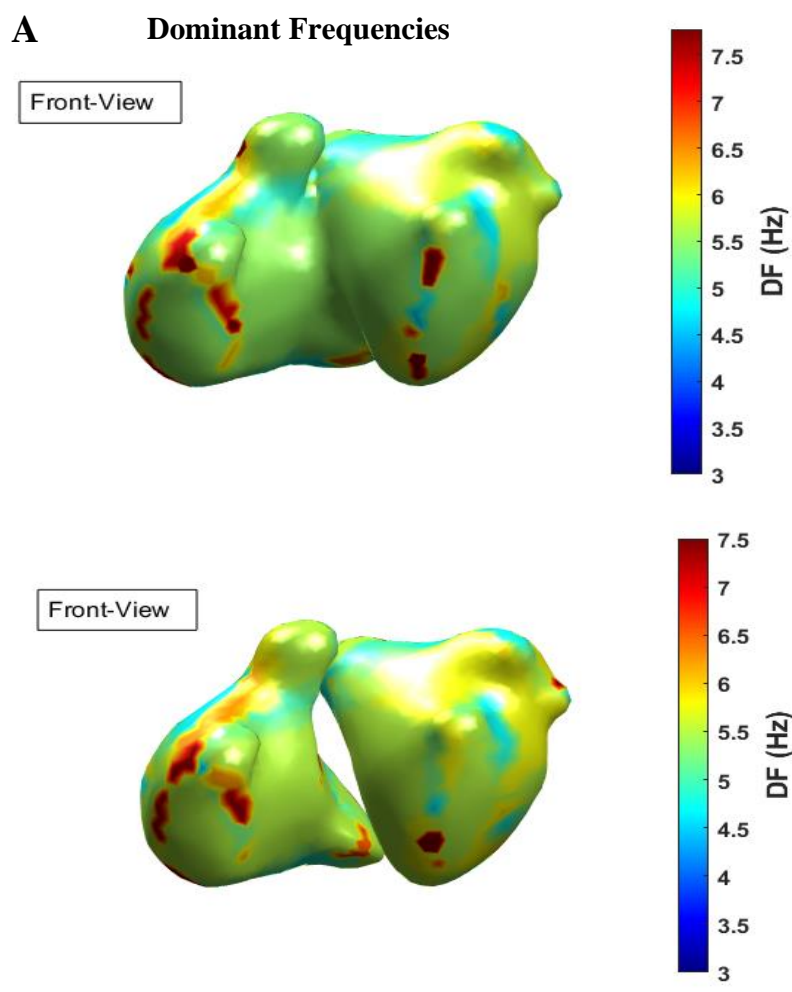

B Rotor Histogram
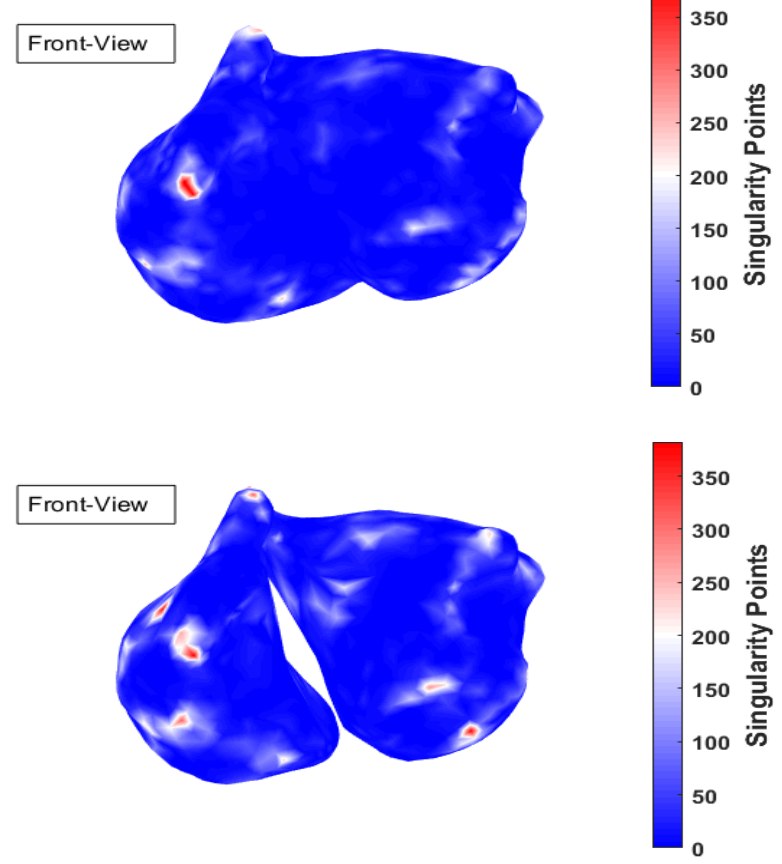

Figure 2. A. Frequency Histogram for a patient where the dominant frequencies are represented. B. Rotor histogram for the same patient where the amount of singular points is showed. 


\section{Conclusions}

Our results show that the consequences if two volumes are segmented for the atria instead of one are only representative for the rotor related analysis while the results obtained for the dominant frequency metrics continue unchanged. Both metrics and maps analysis support the same conclusion. Because of that and due to the importance of these results when used in a procedure, a joined atria segmentation seems to be more desirable.

\section{Acknowledgments}

Supported in part by: Instituto de Salud Carlos III FEDER (Fondo Europeo de Desarrollo Regional PI17/01106), Ministerio de Economía y Competitividad (PEJ2018003617-A) and EIT Health (Activity code 19600). EIT Health is supported by EIT, a body of the European Union

\section{References}

[1] D. B. Geselowitz, "On Bioelectric Potentials in an Inhomogeneous Volume Conductor," Biophys J., vol. 7, no. 1, pp. 1-11, Jan.1967.

[2] M. Rodrigo et al., "Solving Inaccuracies in Anatomical Models for Electrocardiographic Inverse Problem Resolution by Maximizing Reconstruction Quality," IEEE Tran. Med. Imaging, vol. 37, no. 3, pp. 733-740, March 2018.

[3] M. Rodrigo et al., "Noninvasive Assessment of Complexity of Atrial Fibrillation, Correlation with Contact Mapping and Impact of Ablation", Circ. Arrhy. Electrophysiol., vol. 13, No. 03, Feb. 2020.

[4] F. Castells, C. Mora, J. J. Rieta, D. Moratal-Pérez, and J. Millet, "Estimation of Atrial Fibrillation Wave from Singlelead Atrial Fibrillation Electrocardiograms Using Principal Component Analysis Concepts,", Med. Biol. Eng. Comput.., vol. 43, no. 5, pp. 557-560, 2005.

[5] M. Rodrigo et al., "Highest Dominant Frequency and Rotor Positions are Robust Markers of Driver Location during Noninvasive Mapping of Atrial Fibrillation: A Computational Study,", Heart Rhythm, vol. 14, no 8, pp. 1224-1233, Apr. 2017.

Address for correspondence:

Ana González Ascaso.

Instituto Universitario de Tecnologías de la Información y Comunicaciones

Universitat Politécnica de Valencia.

Camino de Vera, s/n, 46022 Valencia, Spain

angonas1@itaca.upv.es 